Discussion Studies show that there is a high prevalence of MSDs among dental professionals all over the world, which is related to their work environment, individual characteristics of the worker and the ergonomics of dental practice. There are many opportunities to assist dental professionals, particularly the dental technicians, to maintain optimal health during the course of their career. This could be done through improved occupational health practice, education on risk factors for MSDs, adjustment of the work environment and wellness maintenance. These measures will improve the quality of life, and ensure that older technicians do not leave the profession due to occupational injuries.

\section{ANXIETY AMONG HEALTH CARE WORKERS OF A TEACHING HOSPITAL}

R Pougnet, G Verdier, P Saliou, F Dantec, B Eniaffe, Q Durand-Moreau, B Loddé, JD Dewitte. Teaching Hospital Of Brest, Brest, France

\subsection{6/oemed-2018-ICOHabstracts.399}

An investigation in psychological suffering in all hospital workers of a teaching hospital has been conducted during one month.

Population and methods Our population was composed of 5792 non medical professionals of a teaching universitary hospital. 1738 workers were randomly selected, and answered an online auto-questionnaire, consisting of 4 parts: socio-professional data; JCQ questionnaire (Karasek); HAD questionnaire and working experience scale. Data were collected on Sphinx Online R software and exploited on Excel, using Chi2 statistical test with $\mathrm{p}<0.05$.

Results In this study, 822 questionnaires were exploitable, representing $47.3 \%$ of the population, and including $81.3 \%$ of women. The mean age was 41.8 years old, and workers were $84.3 \%$ permanent staff. Job strained staff represented $51.7 \%$ of the population, whereas $33.7 \%$ of the staff were in active. The overall isostrain was $31.6 \%$. More permanent workers, nurse's aide and hospital keener were job strained $(\mathrm{p}<0.05)$. Depression prevalence was of $8 \%$ : men, technical agents and people between 42 and 50 years old had a higher risk of depression, respectively 13\%, 15.3\% and $13.4 \% \quad(\mathrm{p}<0.05)$. Anxiety prevalence was of 21.6\%: agents before 42 and 50 years old, permanent workers, nurse's aide, had an increased risk of presenting anxious disorder, respectively 28.2\%, 22.9\% and $28 / 3 \%(\mathrm{p}<0.05)$.

Conclusion Anxiety and depression prevalences were important in this study, in a hospital environment, and were higher than in general population. High level of psychological demand partly explained those prevalences.

\section{WORK-RELATED VIOLENCE AMONG YOUNG NIGHT WORKERS IN SOUTH KOREA}

\footnotetext{
${ }^{1}$ Jia Ryu*, ${ }^{2}$ Hyunjoo Kim, ${ }^{3}$ Jaemin Kim, ${ }^{1}$ Yeogyeong Yoon, ${ }^{1}$ Kyunghee Jung-Choi. ${ }^{1}$ Department of Occupational and Environmental Medicine, College of Medicine, Ewha Womans University, South Korea; ${ }^{2}$ Department of Occupational and Environmental Medicine, Ewha Womans University Mokdong Hospital, South Korea; ${ }^{3}$ Seoul Labour Centre, South Korea
}

10.1136/oemed-2018-ICOHabstracts.400
Introduction In the 24 hour society, night workers encounter more chance of violence. This study aimed to investigate the prevalence of work-related violence and the mental health problems related to the work-related violence of young adults who worked as night workers in South Korea.

Methods The survey was conducted among 507 night workers aged 19-29 who worked in convenience stores, coffee shops, and fast-food restaurants in Seoul, South Korea. The data were obtained by well-trained interviewers with a structured questionnaire. Work-related violence was defined as a physical attack, verbal abuse, sexual harassment, and bullying that occurred in work-related situations or circumstances. The risks of post-traumatic stress disorder, insomnia, and depression were measured using the impact event scale-revised scores, insomnia severity index, and PHQ-9, respectively.

Results Among total 507 night workers included in the study, 159 (31.4\%) had experienced at least one violent incident during their work hours. Among workers who had experienced violence in the workplace, verbal abuse was the most common type. The prevalence of work-related violence increased with longer night working time and longer tenure. Regardless of the type of violence, most of the perpetrators were found to be customers. We observed that $27(17.0 \%)$ of the workers who had experienced violence were at high risk of post-traumatic stress disorder (PTSD). Furthermore, the night workers who had experienced sexual harassment and bullying were shown to have a $41 \%$ and $67 \%$ higher risk of PTSD, respectively. In addition, we found that night workers who had experienced work-related violence were subject to 3.0 and 3.2 times increased risk of insomnia (95\% CI: 1.98 to 4.62 ) and depression (95\% CI: 1.91 to 5.38), respectively.

Conclusion Work-related violence which night workers experienced could be a risk factor for mental illness. Protective intervention against violence toward night workers is urgently needed.

\section{THE INFLUENCE OF WORKING HOURS ON DEPRESSIVE SYMPTOMS IN SOUTH KOREA}

${ }^{1}$ Yeogyeong Yoon*, '1ia Ryu, ${ }^{2}$ Hyunjoo Kim, ${ }^{1}$ Kyunghee Jung-Choi. 'Department of Occupational and Environmental Medicine, College of Medicine, Ewha Womans University, South Korea; ${ }^{2}$ Department of Occupational and Environmental Medicine, Ewha Womans University Mokdong Hospital, South Korea

\subsection{6/oemed-2018-ICOHabstracts.401}

Introduction South Korea is one of the countries with the longest working hours in the OECD countries. Long working hours can be a risk factor of mental illness as well as cardiovascular disease. The aim of this study was to evaluate the effect of long working hours on depressive symptoms among employees in South Korea.

Methods This study used data from the Korea Working Conditions Survey in 2014. Study subjects included 22910 employees aged 19 years or older who work more than 35 hours per week. Working hours were categorised into 35$40,41-52,53-68$, and more than 68 hours per week. Depressive symptoms were assessed using the WHO's Well-Being Index with cut-off score of 50. We calculated prevalence ratios of depressive symptoms according to working hours using log-binomial regression.

Results Only 38.6\% of Korean employees worked 35-40 hours per week. The proportion of employees who worked 41-52, 53-68, and more than 68 hours were 32.7, 15.4, and 4.7\%, 
respectively. The rate of depressive symptoms was $42.8 \%$, and the average of well-being index in Korea was 58.3. The longer the working hours were, the higher the risk of depressive symptoms was. Even after adjusting for sex, age, socioeconomic position, physical problems, job stress and exposure to violence, the risks of depressive symptoms were significantly higher in people who worked 53-68 hours/week (PR, 1.13 95\% CI: 1.09 to 1.17 ), and more than 68 hours/week (PR, 1.07 95\% CI: 1.02 to 1.13 ) than 35-40 hours/week.

Conclusion We showed the long working hours could be an independent risk factor for depressive symptoms in employees. Long working hours can lead to the lack of time to recover and sleep disturbances, which can be associated with depressive symptoms. It is necessary to adjust to the appropriate working hours for employees' mental health.

\section{THE 2016 GLOBAL BURDEN OF DISEASE ARISING FROM OCCUPATIONAL EXPOSURES}

Tim Driscoll. Sydney School of Public Health, University Of Sydney, Sydney, Australia

\subsection{6/oemed-2018-ICOHabstracts.402}

Introduction There are a variety of hazards faced by workers across the globe. Many are common to workers in similar occupations or industries in different regions. Others comprehensive study of the occupational burden of disease has been most recently studied as part of the Global Burden of Disease (GBD) study, headed by the Institute of Health Metrics and Evaluation at the University of Washington.

Methods The risk factors section of the GBD study uses a population attributable fraction (PAF) approach, combining relative risk estimates from the published literature with estimates of exposure prevalence. The PAFs are applied to estimates of the total number of cases or Disability Adjusted Life Years (DALYs) of a particular disorder to produce estimates of the number of deaths or the overall burden (in DALYs) arising from individual occupational risk factors and groups of risk factors. The included risk factors are a range of carcinogens resulting in cancer; particulate matter, gases and fumes resulting in chronic obstructive lung disease; asthmagens causing asthma; noise causing noise-induced hearing loss; ergonomic risk factors causing low back pain; and injury risk factors resulting in injury.

Results The preliminary results for 2016 suggest that the largest burden in terms of deaths was from carcinogens, injury risk factors and particulate matter, gases and fumes. In terms of DALYs, the largest burden was from ergonomic factors, injury risk factors, particulate matter, gases and fumes and noise. The rates and PAFs varied by region and across age and gender.

Conclusion Studying the burden of disease arising from occupational exposures provides guidance to policy makers and practitioners regarding the scope of ill health resulting from work and where resources might most be needed.

\section{NEW EU-OSHA-APPROACH TO QUANTIFY EXPOSED WORKER POPULATIONS IN THE EU}

L Lieck*. European Agency for Safety and Health at Work, Bilbao, Spain

10.1136/oemed-2018-ICOHabstracts.403
Introduction The quantification of the number of workers that are exposed to dangerous substances is a key element for evidence based policy decisions, particularly in the area of occupational safety and health legislation and regulation of chemicals in general. In the past three decades a lot of scientific efforts were made to quantify the number of exposed workers at national, at EU and at international level. The methodological difficulties can be categorised in three major topics:

- The availability of reliable and representative exposure data

- The availability of substance and sector specific production and usage data

- The availability of detailed statistics on sectoral employment, occupations and major working tasks.

Methods The EU-OSHA approach is characterised by a combination of production and usage data from PRODCOM, SPIN and ECHA with survey data from the European Working Conditions Survey (EWCS), the Labour Force Survey (LFS) and the European Survey on New and Emerging Risks (ESENER). Moreover, these data are combined with structural business data of EUROSTAT. In a second step the findings are correlated with national datasets, e.g. from follow-up projects of CAREX or national cancer registers. Still the results of this correlation have to be justified by sector experts' assessment. Further datasets at European level (the planned EU OSH InfoSystem) or specific surveys on certain substances will improve the reliability and comprehensiveness.

Results Improved reliability and precision of quantitative assessment of the occurrence of exposure to substances in certain sectors and occupations, and the best possible estimate of the number workers exposed to these substances.

Discussion Public data on chemical substances is constantly increasing and providing more precision. It will be possible to update and improve the figures from previous estimation approach.

\section{ENVIRONMENTAL ASBESTOS EXPOSURE IN SOUTHERN ITALY: MESOTHELIOMA CASES DUE TO THE SAME POLLUTION SOURCE}

${ }^{1} \mathrm{~L}$ Vimercati*, 'D Cavone, 'L De Maria, ${ }^{1} \mathrm{~A}$ Caputi, , $\mathrm{M}$ Quarato, ${ }^{2} \mathrm{G}$ Serio. ${ }^{1}$ Interdisciplinary Department of Medicine, Occupational Medicine 'B.Ramazzini', University of Bari Medical School, Bari, Italy; ${ }^{2}$ Department of Emergency and Organ Transplantation (DETO), Pathology Division, University of Bari Medical School, Bari, Italy

\subsection{6/oemed-2018-ICOHabstracts.404}

Introduction Bari municipality (Apulia region, Southern Italy) has been defined as a contaminated site of national priority for remediation because of diffuse environmental contamination caused by an asbestos cement factory. Up to date, the regional mesothelioma registry listed 66 environmental mesothelioma cases among residents. We report two peculiar cases of malignant mesothelioma, one peritoneal and one pleural, due to residential asbestos exposure close to a military barracks, located inside the urban area and reclaimed only in 2006.

Methods The records of the two cases with environmental exposure were listed in the Apulia regional mesothelioma registry. The registry ensures complete, quality information on exposure and diagnosis thanks to the adoption of operative guidelines agreed at national level. Molecular analysis with CGH-array was performed on paraffin-embedded tissue. 